\title{
Power Allocation Scheme based on System Outage Probability for Energy-Harvesting Cooperative Networks
}

\author{
Wang $\mathrm{Hao}^{1,}$ a , Zhu $\mathrm{Qi}^{2, \mathrm{~b}}$ \\ ${ }^{1}$ The Key Wireless Laboratory of Jiangsu Province, School of Telecommunication and Information \\ Engineering, Nanjing University of Posts and Telecommunications, Nanjing 210003, China \\ ${ }^{2}$ Key Laboratory on Wideband Wireless Communications and Sensor Network Technology of \\ Ministry of Education, Nanjing 210003, China \\ aemail: njuptwh@126.com, bemail: zhuqi@njupt.edu.cn
}

Keywords: Cooperative Communication; Multi-relay; Energy-Harvesting; Outage Probability; Power Allocation

\begin{abstract}
A power allocation scheme is proposed to improve the outage performance for a wireless cooperative network with multiple energy-harvesting relay nodes. The optimization problem is formulated to minimize the system outage probability under the constraints of both relay joint maximum transmit power and individual energy limitation caused by energy-harvesting. The optimal transmit power of multi-relay can be solved by using Lagrange multiplier method. Simulation results show that the proposed power allocation scheme can reduce system outage probability efficiently.
\end{abstract}

\section{Introduction}

Cooperative communication has attracted increasing attention because of its ability to improve the performance of wireless networks efficiently [1]. Energy-harvesting (EH) has been regarded as an advanced technology to supply power for relay nodes, which can collect energy from renewable resources in ambient environment, so that the lifetime of energy-constrained wireless networks would be prolonged by energy-harvesting [2].

Therefore, the combination of cooperative relaying and energy-harvesting has aroused lots of research interest [3][4]. The authors of [5] investigate the power allocation scheme based on a two-hop EH cooperative network with single relay. A power allocation algorithm with the goal of maximizing the system throughput under the scenario of single relay is proposed in [6]. In this paper, both the source node and relay node work under the energy-harvesting model. The author of [7] proposes a power splitting-based relaying protocol for EH system, which the relay nodes harvest energy from the received signals and use it as the transmit power to forward the signals to the destination node. A two-hop relaying system with direct link and multiple energy-harvesting relays is taken into consideration in [8]. After that, the author gives the close-form expressions for the system outage probability, but the relay power allocation problem is not investigated. The authors of [9] consider a system model of two-hop and three nodes, and propose an optimal power allocation scheme of the source node based on minimum outage probability. However, this paper only takes the power allocation of the stable power supply source node into consideration, without the consideration of the power allocation problem of the EH relay node.

In this paper, we consider a two-hop system with multiple energy-harvesting relay nodes and without direct link between source node and destination node. An optimization problem for minimizing system outage probability is modeled under both relay joint maximum transmit power and individual energy constraints. After that, the optimal transmit power of energy-constrained relay nodes is obtained. Simulation results show that the proposed power allocation scheme can reduce system outage probability efficiently.

The rest of this paper is organized as follows: In Section 2, the system model is presented. In Section 3, we formulate the optimization problem to minimize system outage probability and obtain the optimal transmit power of EH relay nodes. Simulation results are presented and analyzed in 
Section 4. Finally, Section 5 concludes the paper.

\section{System Model}

The two-hop multi-relay network model is shown in Figure1, which is consist of one source node, one destination node and $K$ relay nodes, denoted as $S, D$ and $R_{k}(1 \leq k \leq K)$, respectively.

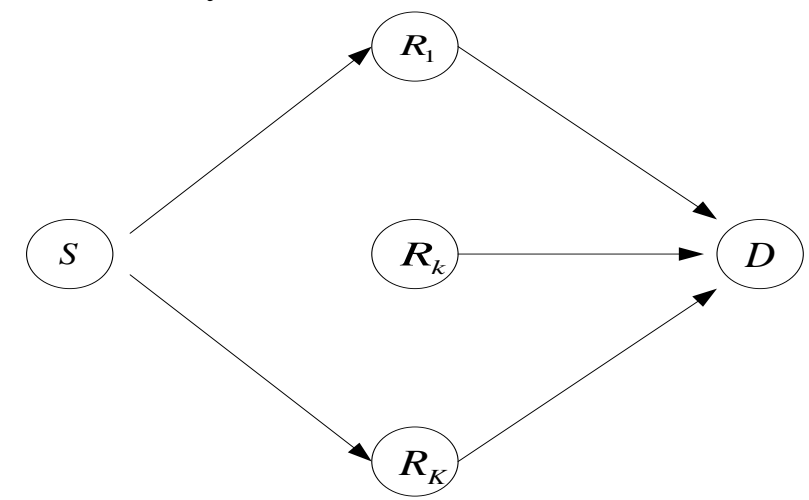

Fig.1. Multi-relay network model

Assuming that there is no direct link between source node and destination node, and $K$ relay nodes help forwarding the transmitted signal from $S$ to $D$. All relay nodes operate in a half-duplex mode and cannot receive and transmit information simultaneously [10]. The communication process between $S$ and $D$ is divided into two phases. In the first phase, source node $S$ broadcasts its information to all relay nodes. In the second phase, $K$ relay nodes amplify the signal received in the first phase and forward it to the destination node $D$. Source node $S$ has a fixed transmit power $P_{s}$ and powered by traditional stable way. Each relay node $R_{k}(1 \leq k \leq K)$ is equipped with energy-harvesting device and collects energy from the received signal.

The received signal at the relay nodes in the first phase and at the destination node in the second phase can be expressed respectively as

$$
\begin{aligned}
& y_{s, r_{k}}=\sqrt{P_{s}} h_{s, r_{k}} x+n_{s, r_{k}} ; \quad \forall k=1,2, \ldots, K \\
& y_{r_{k}, d}=\beta_{r_{k}} \sqrt{P_{r_{k}}} h_{r_{k}, d} y_{s, r_{k}}+n_{r_{k}, d} ; \quad \forall k=1,2, \ldots, K
\end{aligned}
$$

where $x$ is the data symbol to be transmitted, $P_{s}$ and $P_{r_{k}}$ are the transmit power of source node $S$ and relay node $R_{k}, \beta_{r_{k}}$ is the power amplification factor of relay nodes and is given by

$$
\beta_{r_{k}}=\sqrt{\frac{1}{\left(P_{s}\left|h_{s, r_{k}}\right|^{2}+\sigma_{s, r_{k}}^{2}\right)}}
$$

$h_{s, r_{k}}$ and $h_{r_{k}, d}$ are the channel gain of the link $S-R_{k}$ and the link $R_{k}-D$, respectively. They are modeled as zero-mean complex Gaussian random variables with variances $\sigma_{s, r_{k}}^{2}$ and $\sigma_{r_{k}, d}^{2}$. The $n_{s, r_{k}}$ and $n_{r_{k}, d}$ are additive white Gaussian noise (AWGN) with variances $N_{s, r_{k}}$ and $N_{r_{k}, d}$. For simplicity, we assume that $N_{s, r_{k}}=N_{r_{k}, d}=\sigma_{0}^{2}, \forall k \in K \quad[11]$.

The destination node uses maximal ratio combiner to combine the signals received from the $K$ relay nodes [12], and then the total signal to noise ratio (SNR) $\gamma$ at destination node $D$ can be expressed as

$$
\gamma=\sum_{k=1}^{K} \gamma_{r_{k}}=\sum_{k=1}^{K} \frac{\frac{P_{s}}{\sigma_{0}^{2}}\left|h_{s, r_{k}}\right|^{2} \frac{P_{r_{k}}}{\sigma_{0}^{2}}\left|h_{r_{k}, d}\right|^{2}}{\frac{P_{s}}{\sigma_{0}^{2}}\left|h_{s, r_{k}}\right|^{2}+\frac{P_{r_{k}}}{\sigma_{0}^{2}}\left|h_{r_{k}, d}\right|^{2}+1}
$$

According to [8], the system outage event in energy-harvesting condition can be caused by two aspects, one is that the energy which is harvested by relay node $R_{k}$ is not enough to forward the 
information, another is the channel condition of $S-R_{k}-D$ is poor.

The outage probability of channel $S-R_{k}-D$ can be expressed as

$$
\begin{aligned}
P_{s, r_{k}, d}^{\text {out }} & =P\left\{C_{s, r_{k}, d}<R_{0}\right\} \\
& =P\left\{\frac{1}{2} \log \left(1+\gamma_{r_{k}}\right)<R_{0}\right\} \\
& =P\left\{\gamma_{k}<2^{2 R_{0}}-1\right\} \\
& \approx\left(\frac{\sigma_{0}^{2}}{\sigma_{s, r_{k}}^{2} P_{s}}+\frac{\sigma_{0}^{2}}{\sigma_{r_{k}, d}^{2} P_{r_{k}}}\right)\left(2^{2 R_{0}}-1\right)
\end{aligned}
$$

where $R_{0}$ is the required transmission rate, the factor $1 / 2$ is included because the signal is transmitted in two time slots.

Assuming that there is no direct link between source node and destination node, the expression of outage probability for energy-harvesting system is given by

$$
P_{c o}^{\text {out }}=\prod_{k=1}^{K}\left[p_{k}^{\text {ex }}+\left(1-p_{k}^{\text {ex }}\right) \cdot P_{s, r_{k}, d}^{\text {out }}\right]
$$

where $p_{k}^{e x}$ is called as energy-exhausted probability of relay node $R_{k}$, which means $R_{k}$ cannot harvest enough energy to support the communication process.

Substituting Eq. (5) into Eq. (6), we have [8]

$$
P_{c o}^{\text {out }}=\prod_{k=1}^{K}\left\{p_{k}^{e x}+\left(1-p_{k}^{e x}\right) \cdot\left(\frac{\sigma_{0}^{2}}{\sigma_{s, r_{k}}^{2} P_{s}}+\frac{\sigma_{0}^{2}}{\sigma_{r_{k}, d}^{2} P_{r_{k}}}\right)\left(2^{2 R_{0}}-1\right)\right\}
$$

So the optimization problem to minimize the system outage probability under the assumption of fixed source node transmit power $P_{s}$ can be formulated as

$$
\begin{array}{ll}
\mathrm{P}^{*}=\min _{\mathrm{P}} & P_{c o}^{\text {out }} \\
\text { s.t. } & \sum_{k=1}^{K} P_{r_{k}} \leq P_{\max } \\
& \quad 0 \leq P_{r_{k}} \leq P_{r_{k}}^{e h}, \forall k=1,2, \ldots, K
\end{array}
$$

where $P_{\max }$ is defined as relay joint maximum transmit power, the energy harvested from source node by relay node $R_{k}$ donates as $P_{r_{k}}^{e h}$ [13], which is given by

$$
P_{r_{k}}^{e h}=\frac{\eta P_{s}\left|h_{s, r_{k}}\right|^{2}}{d_{s, r_{k}}^{2}} \cdot T
$$

where $0<\eta \leq 1$ is the energy conversion efficiency, $d_{s, r_{k}}$ denotes the distance between source node $S$ and relay node $R_{k} . T$ is the duration of a single time slot and can be normalized to 1 , thus the power and the energy refer to a same meaning in this paper, namely, $P_{r_{k}}^{e h}=\eta P_{s}\left|h_{s, r_{k}}\right|^{2} / d_{s, r_{k}}^{2}$.

\section{Power Allocation Scheme}

An optimal power allocation scheme based on minimum outage probability is proposed in this section. According to Eq. (7) and Eq. (8), and without loss of generality, assuming that the energy-exhausted probability of every relay node is equal, namely, $p_{k}^{e x}=p_{0}, \forall k=1,2, \ldots, K$. Then the optimization problem of Eq. (8) can be simplified as 


$$
\begin{array}{ll}
\mathrm{P}^{*}=\min _{\mathrm{P}} \prod_{k=1}^{K}\left(\frac{1}{\sigma_{s, r_{k}}^{2} P_{s}}+\frac{1}{\sigma_{r_{k}, d}^{2} P_{r_{k}}}\right) \\
\text { s.t. } \quad \sum_{k=1}^{K} P_{r_{k}} \leq P_{\max } \\
& 0 \leq P_{r_{k}} \leq P_{r_{k}}^{e h}, \quad \forall k=1,2, \ldots, K
\end{array}
$$

Apply $\ln (\cdot)$ function to the objective function, and the objective function can be rewritten as

$$
f\left(P_{r_{k}}\right)=\sum_{k=1}^{K} \ln \left(\frac{1}{\sigma_{s, r_{k}}^{2} P_{s}}+\frac{1}{\sigma_{r_{k}, d}^{2} P_{r_{k}}}\right)
$$

From Eq. (7), we can see that the minimum outage probability can be obtained when $\sum_{k=1}^{K} P_{r_{k}}=P_{\max }$, otherwise we can continue to increase the transmit power to further reduce the outage probability. So the Lagrange function of the optimization problem can be given by

$$
L\left(P_{r_{k}}, \lambda\right)=\sum_{k=1}^{K} \ln \left(\frac{1}{\sigma_{s, r_{k}}^{2} P_{s}}+\frac{1}{\sigma_{r_{k}, d}^{2} P_{r_{k}}}\right)+\frac{1}{\lambda}\left(\sum_{k=1}^{K} P_{r_{k}}-P_{\max }\right)
$$

Note that the Lagrange multiplier $1 / \lambda$ is selected to make the calculation and expression more convenient later in this paper.

Take partial derivation of Eq. (12) with respect to $P_{r_{k}}$, and make them equal to zero, then we get

$$
\frac{\partial L\left(P_{r_{k}}, \lambda\right)}{\partial P_{r_{k}}}=-\frac{\sigma_{s, r_{k}}^{2} P_{s}}{\sigma_{r_{k}, d}^{2} P_{r_{k}}+\sigma_{s, r_{k}}^{2} P_{s}} \cdot \frac{1}{P_{r_{k}}}+\frac{1}{\lambda}=0 ; \forall k=1,2, \ldots, K
$$

In order to solve Eq. (13), we define

$$
\mathbf{b}=\left[\frac{\sigma_{r_{1}, d}^{2}}{\sigma_{s, r_{1}}^{2}}, \frac{\sigma_{r_{2}, d}^{2}}{\sigma_{s, r_{2}}^{2}} \cdots \frac{\sigma_{r_{K}, d}^{2}}{\sigma_{s, r_{K}}^{2}}\right]
$$

So we have

$$
b_{k} P_{r_{k}}^{2}+P_{s} P_{r_{k}}-\lambda P_{s}=0, \forall k=1,2, \ldots, K
$$

There are two solutions of Eq. (15), as one of the solutions is negative, so it can be ignored and the $P_{r_{k}}$ can be written as

$$
P_{r_{k}}=\frac{\sqrt{P_{s}^{2}+4 b_{k} P_{s} \lambda}-P_{s}}{2 b_{k}}
$$

where $\lambda$ is a constant which is selected to meet the relay joint maximum transmit power constraint. It can be obtained by using golden section method.

According to KKT (Karush-Kuhn-Tucker) conditions, when the outage probability get the minimum value, if $P_{r_{k}}>P_{r_{k}}^{e h}$, the optimal power allocation must be obtained in the border, namely, $P_{r_{k}}=P_{r_{k}}^{e h}$. So the optimal transmit power of relay $R_{k}$ is given by

$$
P_{r_{k}}^{*}=\min \left(\frac{\sqrt{P_{s}^{2}+4 b_{k} P_{s} \lambda}-P_{s}}{2 b_{k}}, P_{r_{k}}^{e h}\right)
$$

The optimal power allocation scheme can be summarized by the following steps:

1) Initialize the counter variable $k$ as $k=1$.

2) Calculate the harvested energy value $P_{r_{k}}^{\text {eh }}$ of relay node $R_{k}$ according to Eq. (9).

3) Calculate the allocated power value $P_{r_{k}}$ of relay node $R_{k}$ according to Eq. (16).

4) Take the smaller value in $P_{r_{k}}^{e h}$ and $P_{r_{k}}$ as the optimal transmit power $P_{r_{k}}^{*}$ of relay node $R_{k}$. 
5) $\quad k=k+1$, if $k>K$, go to 6); otherwise, go to 2).

6) Obtain the optimal power allocation scheme.

\section{Simulation Results and Analysis}

The simulation parameters in this section are set just as in [14]. The required transmission rate is set as $R_{0}=1$, the distance between source node $S$ and destination node $D$ is 2 . The relay nodes are uniformly distributed in the circle around the midpoint of $S$ and $D$ with radius 2. The channels between source node and relay nodes as well as relay nodes and destination node are modeled as Rayleigh fading channels. The channel coefficients are modeled as complex Gaussian variables, that is $h_{i, j} \sim \mathcal{C N}\left(0,1 / d_{i, j}^{v}\right)$, where $d$ is the distance between node $i$ and node $j$, $v=3$ stands for the distance attenuation factor.

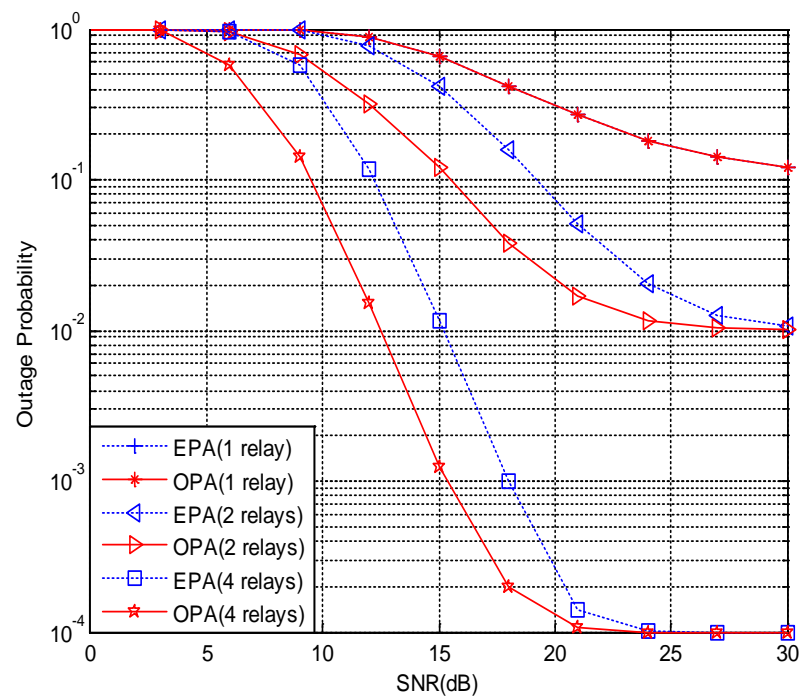

(a) The comparison between EPA and OPA

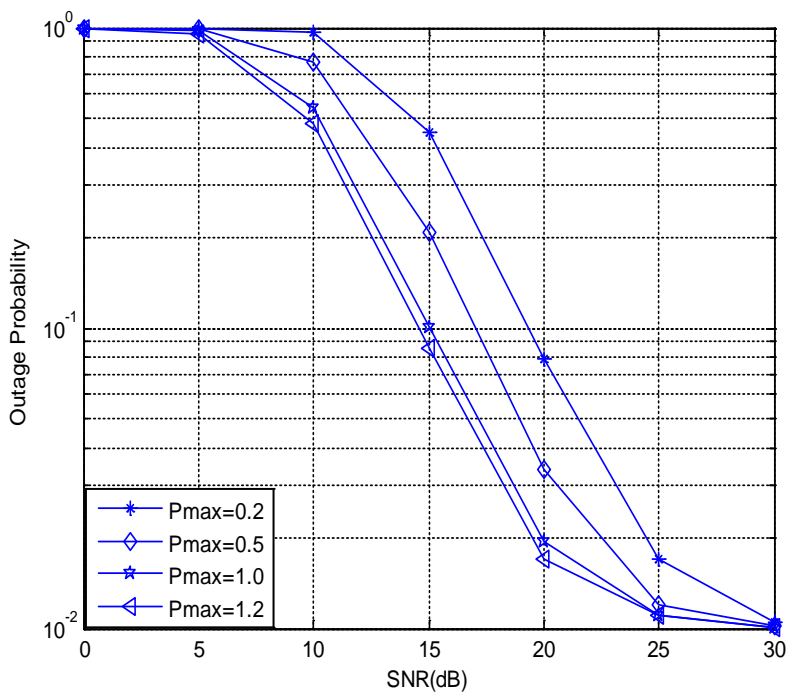

(b) The comparison among different $P_{\max }$

Fig.2. The simulation results

Figure2 (a) shows the comparison of the outage probability between equal power allocation (EPA) scheme and proposed optimal power allocation (OPA). Assuming that there are $K=1,2,4$ relay nodes, the energy-exhausted probability of each relay node is equal, and that is $p_{k}^{e x}=0.1, \forall k=1,2, \ldots, K$, the energy conversion efficiency is $\eta=0.5$. It can be observed from Figure2 (a) that in medium SNR region, the proposed optimal power allocation scheme can achieve about $3 \mathrm{~dB}$ performance gain against the equal power allocation scheme. In high SNR region, with the change of the number of relay nodes, the outage probability close to $10^{-1}, 10^{-2}$ and $10^{-4}$, respectively.

The outage performances of different maximum transmit power $P_{\max }$ of the relay system are compared in Figure2 (b). Assuming that there are $K=2$ relay nodes and $P_{\max }$ is set as $0.2,0.5$, 1.0 and 1.2, respectively. It is obvious that when $P_{\max }$ is small, the performance gain can be improved in a certain degree through increasing $P_{\max }$ appropriately. But with the continued to increase the transmit power, there is hardly any improvement of the performance gain. This is because the transmit power of the energy-harvesting relay nodes is limited by the harvested energy. So if only increase $P_{\max }$ unilaterally, the improvement of the performance gain is very little.

\section{Conclusions}

In this paper, the two-hop and AF-based scenario with multiple energy-harvesting relay nodes and without direct link from source node to destination node is discussed. We propose an optimal power allocation scheme to minimize the system outage probability. The simulation results show 
that the proposed OPA scheme is superior to the EPA scheme on the performance, and the performance gain can be improved in a certain degree through increasing the joint maximum transmit power appropriately.

\section{Acknowledgment}

This work is supported by National Natural Science Foundation of China (61571234), National Basic Research Program of China (973 program: 2013CB329005), 863 Program (2014AA01A705).

\section{References}

[1] Lu H C, Liao W J. Cooperative strategies in wireless relay networks [J]. IEEE Journal on Selected Areas in Communications, 2012 30(2) 323-330.

[2] Tutuncuoglu K, Varan B, YenerA. Optimum transmission policies for energy harvesting two-way relay channels. IEEE Int. Conf. Communications [C]. New Jersey: IEEE, 2013. 586-590.

[3] Zhang R, HoCK. MIMO broadcasting for simultaneous wireless information and power transfer [J]. IEEE Trans. Wirel. Commun, 201312 (5) 1989-2001.

[4] Medepally B, Mehta NB. Voluntary energy harvesting relays and selection in cooperative wireless networks [J]. IEEE Trans. Wirel. Commun, 20109 (11) 3543-3553.

[5] O. Orhan, E. Erkip. Optimal transmission policies for energy harvesting two-hop networks. 2012 Conf. Inform. Sciences and Systems [C]. New Jersey: IEEE, 2012. 1-6.

[6] Huang C, Zhang R, Cui S. Delay-constrained Gaussian relay channel with energy harvesting nodes. 2012 IEEE Intl. Conf. on Communications (ICC 2012) [C]. New Jersey: IEEE, 2012. 2433-2438.

[7] Nasir AA, Zhou X, Durrani S, Kennedy RA. Relaying protocols for wireless energy harvesting and information processing [J]. IEEE Trans Wirel Commun, 2013 12(7) 3622-3636.

[8] Li T, Fan P G, et al. Outage Probability of Energy Harvesting Relay-aided Cooperative Networks over Rayleigh Fading Channel [J]. IEEE Transactions on Vehicular Technology, 201599 1.

[9] Li J B, Xi L, et al. Source Nodes Power Optimization in Energy Harvesting Two-Way Relay Networks. The 16th International Conference on Advanced Communications Technology [C]. New Jersey: IEEE, 2014. 584-588.

[10]Bao V, Duong T, Costa D. Cognitive amplify-and-forward relaying with best relay selection in non-identical rayleigh fading[J]. IEEE Communications Letters, 2013 17(3) 475-478.

[11]Zheng B. Outage Probability of Two-Hop Relay Networks with Related Interference [J]. KSII Transactions on Internet and Information Systems (TIIS), 2013 7(8) 1786-1804.

[12] Seddik K G, Sadek A K, Su W. Outage analysis and optimal power allocation for multi node relay networks [J]. IEEE Signal Processing Letters, 2007 14(6) 377-380.

[13]M. Majid Butt, Adnan Nasir, et al. Trading Wireless Information and Power Transfer: Relay Selection to Minimize the Outage Probability. Signal and Information Processing (GlobalSIP) [C]. New Jersey: IEEE, 2014. 253-257.

[14]Zhou Z K, Zhu Q. Joint power allocation and multi-relay selection scheme based on system outage probability [J]. The Journal of China Universities of Posts and Telecommunications, 2014 5(3) 9-16. 